\title{
Perinatal microbiota: review of its importance in newborn health
}

\author{
José Uberos, M.D.a
}

\begin{abstract}
The use of metagenomics in the study of gut bacterial ecosystems has helped to define a standard, functional genetic profile in newborn infants, so that a bacterial ecosystem will be deemed more "normal" the more similar its functional genetic profile is to a standard. The development of a specific functional enterotype in the first days of life after birth is critical for the priming of the immune system with certain bacterial antigens.

Regardless of whether the first gut bacteria are acquired before or just after birth, the newborn microbiota will result from the symbiosis with the environmental microbial flora, especially with the bacterial flora of the mother. The type of delivery, the administration of perinatal antibiotics, the environment, and nutritional exposure, especially breastfeeding, have demonstrated an important relationship with the prevalent gut microbiome.

Key words: microbiota, newborn infant, microbiome, enterotype.
\end{abstract}

http: / / dx.doi.org/10.5546/ aap.2020.eng.e265

To cite: Uberos J. Perinatal microbiota: review of its importance in newborn health. Arch Argent Pediatr 2020;118(3):e265-e270.

\section{INTRODUCTION}

The first notes on the taxonomic classification of life were published in 1753 by Swedish botanist Carl Linnaeus in his Species plantarum.

a. Neonatal Unit, Hospital Universitario San Cecilio, Granada, Spain.

E-mail address: José Uberos, M.D.: juberos@ugr.es

Funding:

None.

Conflict of interest:

None.

Received: 5-3-2019

Accepted: 10-17-2019 based on three domains (Bacteria, Archaea, and Eukarya) (Figure 1). ${ }^{1}$

Woese described the existence of a common ancestor about which there is no valid information and from which the three domains mentioned above derive as a result of their adaptation to the environment. ${ }^{2}$

The set of microorganisms that are normally located in several sites of a multicellular organism is defined as microbiota. The set of genes carried by all microorganisms included in the microbiota is the microbiome, which is the basis for the metagenomic studies that will be explained below. The term metagenomics was first used by Robert M. Goodman ${ }^{3}$ in 1998 to refer to an original approach to genetic testing by treating the set of sequenced genes in a sample as if it were a single genome. Metagenomics may also refer to the set of genomic techniques used to study microbial communities in their natural environment, thus preventing isolation and culture of each species that make up their natural environment. With this technique, research based on the study of the $16 S$ gene as metagenomic using human fecal samples has observed that only 7-9 of the 55 phyla of the Bacteria domain are present. In particular, more than $90 \%$ of the Bacteria domain forms correspond to the Bacteroidetes and Firmicutes phyla. ${ }^{4}$

Among the genes identified in the gut, $98 \%$ are bacterial, and between 1000 and 1150 bacterial species have been described, with a mean of 160 species per individual. As established by the development of the Human Microbiome Project, ${ }^{4}$ microbiome diversity is unique to each individual and strongly related to the microbial habitat. At the gut level, the extent of the bacterial community 
varies depending on the bowel segment under study, from the cecum to the rectum. However, when considered as a whole, the gut flora remains relatively stable. Microbiome stability as a whole appears to be the standard; local changes in the habitat may temporarily alter the bacterial composition but, when considering an individual's body microbiome, it tends to be stable. $^{5}$

Two large projects are currently under way to try and decode the structure and function of the human gut flora and its relation to health and disease status. On the one side, the Metagenomics of the Human Intestinal Tract (MetaHIT) project, funded by the European Union, and on the other side, the Human Microbiome Project, sponsored by the United States National Institutes of Health. The latter has helped to define, to date, 5177 microbial taxonomic profiles with a catalog of 5 million bacterial genes at gastrointestinal level, which code proteins involved in 20000 biological functions.

Undoubtedly, a key aspect to define a healthy bacterial ecosystem is the fact that "in spite of the great inter-individual variability in terms of bacterial taxonomy, the functional genetic profile expressed by the bacterial community is rather similar in healthy individuals". That is to say, the ecosystem will be more "normal" the more similar its functional genetic profile is to a standard. This led to describing the enterotype concept, which may be associated with diet, geographic area, medication use or body habits.
An enterotype is a classification of living organisms based on their bacteriological ecosystem in the gut microbiome. Three basic enterotype profiles have been described, represented by the predominance of a phylum and a bacterial class. ${ }^{6}$ Enterotype 1, the most common one, regardless of the geographic area considered, is related to a protein- and fat-rich diet, and is characterized by the prevalence of Bacteroidetes, Bacteroides class.

Enterotype 2 is related to dysbiosis circumstances with a prevalence of the Bacteroidetes phylum but at the expense of the Prevotella class. Prevotella and Lactobacillus populations are antagonistic, so an increase in Prevotella is associated with a decrease in Lactobacillus. Enterotype 2, specifically the prevalence of Prevotella, has been related to a strict vegetarian diet. Prevotella is a common component of the oropharyngeal microbiome; outside this site, it has been related to vaginal dysbiosis (vaginosis) or chronic bowel inflammation. ${ }^{7}$

Enterotype 3, which is less common than the other two, has been related to a prevalent carbohydrate intake. It has been linked to a prevalence of Ruminococcus (Firmicutes phylum, Clostridia class), a bacterium indicative of dysbiosis, and childhood atopy. ${ }^{8}$

It has been considered that the bacterial density attained by the Firmicutes (Lactobacillus) and Actinobacteria (Bifidobacterium) phyla are critical to warrant an adequate development of the newborn immune system. The development of these two bacterial phyla at the gut helps to

\section{Bacteria Archaea Eukarya}

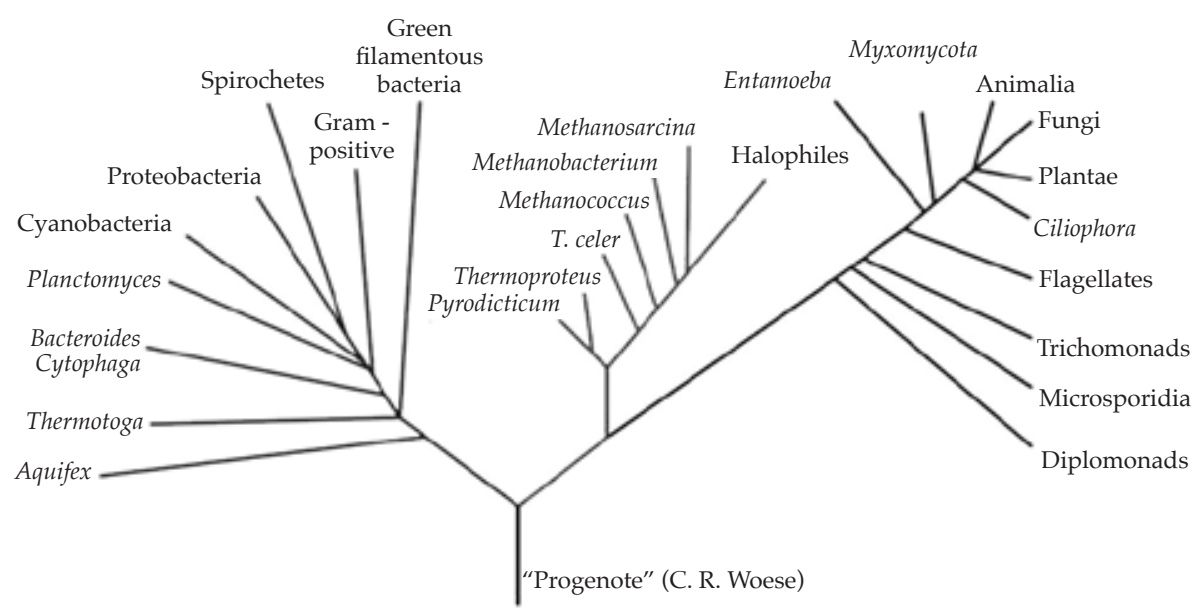


establish adequate conditions for the emergence of the Bacteroidetes phylum and, especially, the Bacteroides class, therefore establishing the enterotype 1.

The type of delivery affects the predominant enterotype development process. Wampach et al., ${ }^{9}$ observed that Firmicutes and Actinobacteria functional pathways, transferred to the newborn infant, are less developed in those born via C-section, while they are over-represented in the bacteria transferred after a vaginal delivery.

The lipopolysaccharide biosynthesis functional pathway, found in the membrane of Gramnegative bacteria, is well represented in the gut microbiome of newborn infants born via vaginal delivery, which promotes the release of proinflammatory cytokines, leading to the priming of the neonatal immune system. Infants born via C-section with a lower lipopolysaccharide content, due to the lower Gram-negative bacterial load, have a smaller immunostimulatory potential at the level of the gut microbiome. Another functional pathway that may interact with the human immune system is the synthesis of cationic antimicrobial peptides (CAMPs). This pathway may evade detection by the human immune system through the modification of the microbial lipopolysaccharide structure and has been identified in all bacteria of the Bacteroidetes phylum. ${ }^{9}$

Based on the foregoing, the optimal functional microbiome in the first days of life may be defined as that made up of a combination of the Firmicutes (Lactobacillus) and the Actinobacteria (Bifidobacterium) phyla, which enable the environmental conditions necessary for the expansion of the Bacteroidetes (Bacteroides) phylum in terms of anaerobiosis. The expansion of a bacterial or other phylum is relevant because it defines the dominant functional pathway and the short-chain fatty acid developed predominately at the gut level. A prevalent Firmicutes phylum is associated with a prevalent butyrate production during the complex carbohydrate fermentation process; whereas the Bacteroidetes phylum is associated with a predominant propionate production.

It is worth noting that butyrate has been described as an agent capable of modifying the histone acetylation state; therefore, it has epigenetic effects, which are currently starting to be understood. ${ }^{10}$

\section{Early bacteria. The dogma of the sterile womb}

Since the beginning of modern perinatology, it has been assumed that both the placenta and the fetus or its environment are sterile and that any alteration in these conditions during gestation may, at times, lead to a preterm birth or the development of chorioamnionitis. ${ }^{11}$ The dogma of the sterile womb takes place in parallel with early $20^{\text {th }}$ century microbiology.

In this period, some investigators found, more than three decades ago, evidence against the fetal-uterine sterility, but the idea that the placenta may have a completely developed microbiome did not gain credibility until 2014, when a research team led by Aagaard identified bacterial deoxyribonucleic acid (DNA) in the placental tissue. ${ }^{12}$ Until then, the isolation of any type of bacteria in placenta or amniotic fluid was necessarily considered an intrauterine infection and, therefore, it was expected to identify the inflammatory processes associated with an increase in acute phase reactants and the concurrent or subsequent development of a clinical infection. The identification of a placental microbiome is not necessarily associated with clinical or analytical signs of infection; sometimes, gestation comes to full term. ${ }^{12}$

These findings, together with the observations by Mysorekar et al., ${ }^{13}$ about the absence of an inflammatory response in the presence of placental bacteria, may suggest that the neonatal microbiome could develop before birth. If this is true, bacteria would be a normal, maybe even critical, part of pregnancy by regulating the developing immune system. ${ }^{13}$

Josef Neu, a neonatologist working at the University of Florida (USA), identified bacterial DNA in the meconium of newborn infants. ${ }^{14}$ According to this investigator, findings seem to confirm that the neonatal microbiome starts developing before birth. He observed a high level of agreement between the identified microbiome in the meconium and that identified in the amniotic fluid. These observations are not exempt from controversy because sterility is lost after the amniotic sac breaks open. Even if a baby is born via C-section, hours or days may pass until the first meconium is produced, which is a large enough time window to acquire bacteria outside the womb. ${ }^{14}$

After birth, the neonatal digestive system goes from a relative intrauterine sterile environment to a dense bacterial colonization in the first few 
weeks of life. Factors like the type of delivery, the administration of perinatal antibiotics, the environment, and nutritional exposure, especially breastfeeding, have demonstrated an important relationship with the prevalent gut microbiome. ${ }^{15}$

After gut colonization, the establishment of the gut flora and the development of the microbiome in neonates and infants are closely related to environmental factors; diet and the nutrients that gut bacteria receive through it are a critical factor. Human milk oligosaccharides are a heterogeneous group with more than 200 compounds described so far. There is a quantitatively higher amount of human milk oligosaccharides than milk proteins, and they account for the element that differentiates human and other animal species milk. Current evidence appears to indicate that protein or fat intake in the mother's diet does not significantly change the proportion of milk macronutrients, although it does alter the proportion of milk oligosaccharides. ${ }^{16,17}$

Ninety-eight per cent of oligosaccharides are not absorbed; they reach the colon, where they are digested by the bifidogenic flora, for which they become a nutritive substrate. Only $1-2 \%$ of the total amount of milk oligosaccharides consumed is absorbed and eliminated in the urine. It has been suggested that the type and amount of maternal milk oligosaccharides determine the type and density of the bifidogenic gut flora.

The breast microbiota starts developing during the last trimester of pregnancy and disappears after weaning. In breastfed infants, the neonatal microbiome is greatly similar to the microbiome observed in the mammary gland which, in turn, is very similar to the mother's gut microbiome. Some studies ${ }^{18}$ appear to indicate that Firmicutes, Bacteroidetes, Actinobacteria, and Proteobacteria are the bacterial phyla usually present in breast milk, with a microbiome that is highly similar to the one observed in the maternal gut. It has been considered that some gut bacteria against which the breastfeeding mother has developed tolerance may be transported in the lymphatic pathway to the mammary gland and may help to develop the mammary gland microbiota. This implies that the infant's microbiota may be regulated through the mother's mammary gland and gut microbiota. ${ }^{18}$

Evolutionary speaking, the microbiota observed in breast milk changes from a very diverse flora identified in the colostrum, where maternal skin and gut bacteria prevail, to a flora identified in the mature milk, where diversity is highly reduced and the infant's mouth and skin bacteria predominate. After weaning, bacterial density decreases drastically until almost disappearing. ${ }^{18}$

Regardless of whether or not prenatal bacterial colonization takes place in the fetus, as suggested by different authors, ${ }^{12,13}$ birth marks the moment of an extensive exposure to fecal, vaginal, skin, and environmental microbial communities, so this event has a deep impact on the newborn's gut colonization. Even aspects related to the type of maternal nutrition, in addition to the type of infant feeding, may affect the neonatal microbiota. ${ }^{19,20}$

The initial microbial biomass is small in the first neonatal fecal samples. With cesarean deliveries, it has been observed that the neonatal gut colonization is delayed and that it coincides with the flora from the mother's skin, whereas in vaginal deliveries, colonization takes place preferably with the flora from the birth canal and the mother's gut. After a cesarean delivery, newborn infants have a smaller proportion of Bacteroides and less Bifidobacteria.

In addition, a different response to humoral immunity has been described in these newborn infants. That is to say, newborn infants born via vaginal or cesarean delivery would have different enterotypes. ${ }^{21}$ If the total number of gut bacteria per gram of feces at the month of life was counted, it would be significantly higher in vaginally-born infants. Such findings have encouraged some investigators to propose a vaginal flora transplant from the mother to infants born via C-section, as a way of "normalizing" the neonatal gut microbiota and, thus, contributing to the maturation of the immune system. 22,23

Since there are no conclusive studies developed with an adequate experimental model, there is controversy about the effectiveness and long-term usefulness of this type of interventions. The initial differences in the neonatal microbiota, depending on the type of delivery, tend to disappear with breastfeeding, especially in the infant once complementary feeding is started..$^{21}$

Another factor that may alter the initial neonatal microbiota is antibiotic use in the mother or newborn infant during the perinatal period. Intrapartum antibiotics are routinely administered to all pregnant women who carry group B Streptococcus. This is referred to as intrapartum antibiotic prophylaxis. Apart from the consequences on the neonatal bacterial flora and microbiome, the benefits of a reduced 
mortality due to neonatal sepsis of vertical transmission caused by group B Streptococcus are evident. $^{24}$

Recent studies have shown, in pregnant women receiving intrapartum prophylaxis with ampicillin, a reduction in the Actinobacteria (Bifidobacterium) phylum in the neonatal fecal flora; however, other bacteria of the Firmicutes phylum, which encompasses Lactobacillus and Clostridia, are not affected. ${ }^{25}$ Changes in the microbiota related to intrapartum antibiotic use are consistent with the antimicrobial spectrum of ampicillin, which fights Gram-positive bacteria, and this would account for the superabundance of Gram-negative bacteria, mainly the Proteobacteria phylum, after antibiotic therapy. ${ }^{25}$

The restoration of the inter-species balance after antibiotic therapy is affected by the spectrum of the antibiotic used. F. Hildebrand et al., ${ }^{26}$ described a situation of monodominance following antibiotic therapy, which is defined as the predominance of a bacterial phylum in more than $60 \%$ of its ecosystem. Borkfalki ceftriaxensis, which is part of the Firmicutes phylum, Clostridia class, predominates in onethird of the population, although it is uncommon at an individual level. Monodominance is a critical step for the expansion of other evolving species in the ecosystem towards stable bacterial communities. This process is related to the fermentation metabolism of $B$. ceftriaxensis that occurs in multiple sources of carbohydrates, which produces short-chain fatty acids that may suppress, among other effects, antibioticassociated diarrhea. In this process, carbohydrate availability at the gut level is critical in the restoration of the gut ecosystem. For this reason, $B$. ceftriaxensis may be used as a probiotic treatment following antibiotic therapy. ${ }^{26}$

Situations that are inherent to maternal health, such as obesity, may also condition microbiota. Obese pregnant women have shown a $50 \%$ reduction in Bacteroides, compared to non-obese controls who had the same diet. Actually, a reduced amount of Bifidobacterium and Bacteroides and a greater number of staphylococci and enterobacteria have been observed in overweight pregnant women. ${ }^{27}$ The larger number of enterobacteria was related to an increase in ferritin and transferrin, whereas more Bacteroides were related to a greater level of high-density lipoproteins (HDL) and folic acid.

Different gut colonization patterns are observed in children from industrialized or developing countries. ${ }^{27}$ In addition, gestational diabetes may cause dysbiosis in the meconial microbiota of newborn infants. Soderborg et al., ${ }^{27}$ observed that Neisseria / Leptotrichia predominated in the maternal oral microbiota and positively related them to blood glucose values, which conditioned the final maternal and neonatal microbiome.

\section{CONCLUSIONS}

The early signs of life on Earth date back more than 3.5 billion years. From then to the present, environmental conditions have made evolution possible, from primitive forms of life to other, more evolved ones, including the human species. In this period, the collaboration among species has been the standard, and the most recent findings appear to indicate that it starts in the perinatal period. Evolution is founded on the achievements made by evolutionary inferior species. Such knowledge underlines the importance of promoting breastfeeding due to its benefits, both nutritional and microbiological, and encouraging a reasonable use of perinatal antibiotic therapy and deliveries by cesarean section, given their consequences on neonatal microbiota.

\section{REFERENCES}

1. Woese CR, KandlerO, Wheelis ML. Towardsa natural system of organisms: proposal for the domains Archaea, Bacteria, and Eucarya. Proc Natl Acad Sci USA. 1990; 87(12):4576-9.

2. WoeseCR. Evolutionary questions: the "progenote". Science. 1990; 247(4944):789.

3. Handelsman J, Rondon MR, Brady SF, Clardy J, et al. Molecular biological access to the chemistry of unknown soil microbes: a new frontier for natural products. Chem Biol. 1998; 5(10):R245-9.

4. Human Microbiome Project Consortium. Structure, function and diversity of the healthy human microbiome. Nature. 2012; 486(7402):207-14.

5. Caporaso JG, Lauber CL, Costello EK, Berg-Lyons D, et al. Moving pictures of the human microbiome. Genome Biol. 2011; 12(5):R50.

6. Arumugam M, Raes J, Pelletier E, Le Paslier D, et al. Enterotypes of the human gut microbiome. Nature. 2011; 473(7346):174-80.

7. Ley RE. Gut microbiota in 2015: Prevotella in the gut: choose carefully. Nat Rev Gastroenterol Hepatol. 2016; 13(2):69-70.

8. Chua HH, Chou HC, Tung YL, Chiang BL, et al. Intestinal Dysbiosis Featuring Abundance of Ruminococcus gnavus Associates With Allergic Diseases in Infants. Gastroenterology. 2018; 154(1):154-67.

9. Wampach L, Heintz-Buschart A, FritzJV, Ramiro-Garcia J, et al. Birth mode is associated with earliest strain-conferred gut microbiome functions and immunostimulatory potential. Nat Commun. 2018; 9(1):5091.

10. Morrison DJ, Preston T. Formation of short chain fatty acids by the gut microbiota and their impact on human metabolism. Gut Microbes. 2016; 7(3):189-200.

11. Uberos J. The significance of genetics in pathophysiologic 
models of premature birth. Minerva Pediatr. 2018; 70(4): 383-90.

12. Aagaard KM, Segars JH. What is the microbiome and how do we study it? Semin Reprod Med. 2014; 32(1):3-4.

13. Mysorekar IU, Cao B. Microbiome in parturition and preterm birth. Semin Reprod Med. 2014; 32(1):50-5.

14. Ardissone AN, De la Cruz DM, Davis-Richardson AG, RechciglKT, etal. Meconium microbiome analysis identifies bacteria correlated with premature birth. PLoS One. 2014; 9(3):e90784.

15. Milani C, Duranti S, Bottacini F, Casey E, et al. The First Microbial Colonizers of the Human Gut: Composition, Activities, and Health Implications of the Infant Gut Microbiota. Microbiol Mol Biol Rev. 2017; 81(4):e00036-17.

16. Hallam MC, BarileD, Meyrand M, GermanJB, etal. Maternal high-protein or high-prebiotic-fiber diets affect maternal milk composition and gut microbiota in rat dams and their offspring. Obesity (Silver Spring). 2014;22(11):2344-51.

17. ChirdoFG, Menendez AM, Pita Martin de Portela ML, Sosa $\mathrm{P}$, et al. Prebióticos en salud infantil. Arch Argent Pediatr. 2011; 109(1):49-55.

18. Latuga MS, Stuebe A, Seed PC. A review of the source and function of microbiota in breast milk. Semin Reprod Med. 2014; 32(1):68-73.

19. Nauta AJ, Ben Amor K, Knol J, Garssen J, et al. Relevance of pre- and postnatal nutrition to development and interplay between the microbiota and metabolic and immune systems. Am J Clin Nutr. 2013; 98(2):586S-93.

20. Toca MdC, Tonietti M, Vecchiarelli C. Nutrición pre- y posnatal: impacto a largo plazo en la salud. Arch Argent
Pediatr. 2015; 113(3):248-53.

21. Stinson LF, Payne MS, Keelan JA. A Critical Review of the Bacterial Baptism Hypothesis and the Impact of Cesarean Delivery on the Infant Microbiome. Front Med (Lausanne). 2018; 5:135.

22. Dominguez-BelloMG, CostelloEK, Contreras M, Magris M, et al. Delivery mode shapes the acquisition and structure of the initial microbiota across multiple body habitats in newborns. Proc Natl Acad Sci U S A. 2010; 107(26):11971-5.

23. Dominguez-Bello MG, De Jesus-Laboy KM, Shen N, Cox LM, et al. Partial restoration of the microbiota of cesareanborn infants via vaginal microbial transfer. Nat Med. 2016; 22(3):250-3.

24. Verani JR, McGee L, Schrag SJ. Prevention of Perinatal Group B Streptococcal Disease-revised guidelines from CDC, 2010. MMWR Recomm Rep. 2010; 59(RR10):1-36.

25. Aloisio I, Quagliariello A, De Fanti S, Luiselli D, et al. Evaluation of the effects of intrapartum antibiotic prophylaxis on newborn intestinal microbiota using a sequencing approach targeted to multi hypervariable $16 \mathrm{~S}$ rDNA regions. Appl Microbiol Biotechnol. 2016;100(12):553746.

26. Hildebrand F, Moitinho-Silva L, Blasche S, Jahn MT, et al. Antibiotics-induced monodominance of a novel gut bacterial order. Gut. 2019; 68(10):1781-90.

27. Soderborg TK, Clark SE, Mulligan CE, Janssen RC, et al. The gut microbiota in infants of obese mothers increases inflammation and susceptibility to NAFLD. Nat Commun. 2018;9(1):4462. 